\title{
Diffusion tensor MRI of the human heart In Vivo with a navigator based free breathing approach
}

\author{
Sonia Nielles-Vallespin ${ }^{1 *}$, Choukri Mekkaoui ${ }^{2}$, Peter D Gatehouse1, Timothy G Reese ${ }^{2}$, Jennifer Keegan', \\ Steven Collins ${ }^{1}$, Peter Speier ${ }^{3}$, Thorsten Feiweier ${ }^{3}$, Marcel P Jackowski ${ }^{4}$, David E Sosnovik ${ }^{2}$, David N Firmin ${ }^{1}$ \\ From 15th Annual SCMR Scientific Sessions \\ Orlando, FL, USA. 2-5 February 2012
}

\section{Summary}

The purpose of this work was to implement prospective navigators to allow free breathing in vivo DTI of the heart to be performed and, thereby, allow the technique to be broadly applied in patients with cardiovascular disease.

\section{Background}

Diffusion tensor MRI (DTI) provides a non-invasive approach for the depiction of the myocardial fibre architecture [1-5]. In vivo DTI remains extremely challenging due to the need for motion correction. Several techniques have been used to compensate for respiratory motion: multiple breath-holds ( $>36$ per patient), synchronised breathing and retrospective navigators based on image cross-correlation [2-5]. The purpose of this work was to implement prospective navigators to allow free breathing in vivo DTI of the heart to be performed and, thereby, allow the technique to be broadly applied in patients with cardiovascular disease.

\section{Methods}

The diffusion weighted (DW) STEAM single shot EPI sequence was implemented on a clinical scanner (3T, MAGNETOM Skyra, Siemens AG, Germany) [7]. The crossed slices prospective navigators were applied before and after the STEAM module. The navigator accept/ reject algorithm was modified to prevent bulk respiratory motion artifacts in the diffusion encoded images. A biofeedback mechanism was implemented to increase scanning efficiency. Eight volunteers were scanned with breath-hold $(\mathrm{BH})$ and navigated free breathing $(\mathrm{FB})$ protocols (6 diffusion encoding directions, $b=350 \mathrm{~s} / \mathrm{mm}^{2}$,

${ }^{1}$ CMR Unit, Royal Brompton And Harefield NHS Foundation Trust, London, UK

Full list of author information is available at the end of the article
$\mathrm{TR} / \mathrm{TE}=1100 / 23 \mathrm{~ms}, \mathrm{BW}=2442 \mathrm{~Hz} /$ pixel, spatial resolution $=2.7 \times 2.7 \times 8 \mathrm{~mm}^{3}, 3$ slices, $8-10$ averages). FA and MD were calculated for 4 sections of the left ventricle (LV) and one of the right ventricle (RV). MD and FA values acquired with the $\mathrm{BH}$ and $\mathrm{FB}$ techniques were compared with a paired t-test.

\section{Results}

Scan duration with the $\mathrm{BH}$ approach was $14.4 \pm 1.5 \mathrm{~min}$ and $17.1 \pm 4.2$ min with the FB approach. The FB approach thus significantly improved volunteer comfort without a major increase in scan duration. An averaged b0 image and FA and MD maps are shown in Figure 1. Figure 2 shows the mean \pm SD of FA and MD values for the different regions of the heart. No significant differences are seen between the $\mathrm{BH}$ and $\mathrm{FB}$ techniques $(\mathrm{p}>0.3)$.

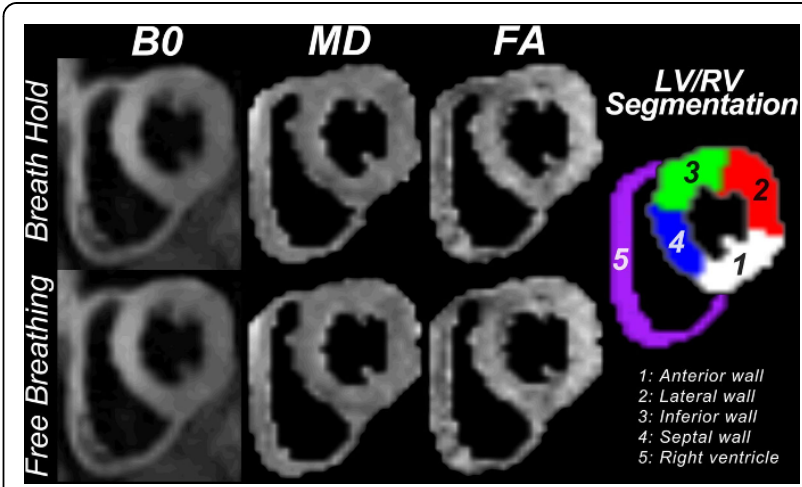

Figure 1 Example b0 image (averaged), FA and MD maps for $\mathrm{BH}$ and FB. 


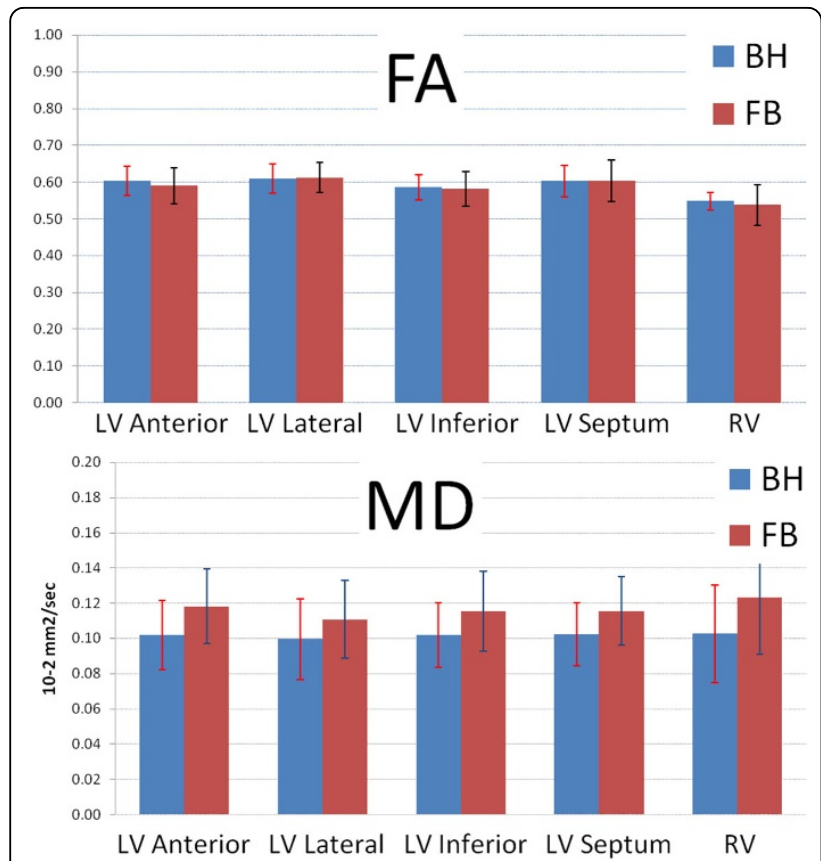

Figure 2 Mean \pm SD of FA and MD values for the different regions of the heart.

\section{Conclusions}

We show here that a free-breathing navigator based approach to DTI produces high quality in vivo images. The ability to perform free breathing DTI will be useful in normal volunteers but critical if the use of DTI is to be extended to patients with cardiovascular disease and limited breath-hold capacity.

\section{Funding}

This project was funded and supported by the NIHR Biomedical Research Unit at the Royal Brompton and Harefield NHS Foundation Trust and Imperial College London, and by the following grant from the National Institutes of Health (R01HL093038).

\section{Author details}

${ }^{1}$ CMR Unit, Royal Brompton And Harefield NHS Foundation Trust, London, UK. ${ }^{2}$ Martinos Center for Biomedical Imaging, Massachusetts General Hospital, Charlestown, MA, USA. ${ }^{3}$ MR R\&D, Siemens AG Healthcare Sector, Erlangen, Germany. ${ }^{4}$ Institute of Mathematics and Statistics, University of São Paulo, São Paulo, Brazil.

Published: 1 February 2012

\section{References}

1. Streeter DD, et al: Circ Res. 1969, 24:339-347.

2. Edelman RR, et al: MRM. 1994, 32:423-428.

3. Reese TG, et al: MRM. 1995, 34:786-791.

4. Dou J, et al: MRM. 2003, 50:107-112.

5. Wu, et al: Circulation. 2006, 114:1036-1045.

6. Gamper U, et al: MRM. 2007, 57:331-337.

7. Nielles-Vallespin, et al: ISMRM. 2011.
doi:10.1186/1532-429X-14-S1-P238

Cite this article as: Nielles-Vallespin et al:: Diffusion tensor MRI of the human heart In Vivo with a navigator based free breathing approach. Journal of Cardiovascular Magnetic Resonance 2012 14(Suppl 1):P238.
Submit your next manuscript to BioMed Central and take full advantage of:

- Convenient online submission

- Thorough peer review

- No space constraints or color figure charges

- Immediate publication on acceptance

- Inclusion in PubMed, CAS, Scopus and Google Scholar

- Research which is freely available for redistribution 